\title{
Comparison of different modalities for the diagnosis of parastomal hernia: a systematic review
}

\author{
Gijs H. J. de Smet ${ }^{1}$ (D) - Daniël P. V. Lambrichts ${ }^{1}$ (D) - Sjoerd van den Hoek ${ }^{2} \cdot$ Leonard F. Kroese $^{3}$ (D) - Stefan Buettner ${ }^{1}$. \\ Anand G. Menon ${ }^{2}$. Gert-Jan Kleinrensink ${ }^{4}$. Johan F. Lange ${ }^{1,2}$
}

Accepted: 23 December 2019 / Published online: 7 January 2020

(C) The Author(s) 2020

\begin{abstract}
Purpose Parastomal hernia (PSH) is a common complication following stoma formation. The incidence of PSH varies widely due to several factors including differences in diagnostic modality, observer, definition, and classification used for diagnosing PSH. The aim of this systematic review was to evaluate the diagnostic accuracy of the modalities used to identify PSH.

Methods Embase, MEDLINE, Cochrane, Web of Science, and Google Scholar databases were searched. Studies reporting PSH incidence rates detected by two or more different diagnostic modalities or inter-observer variation on one diagnostic modality were included. Article selection and assessment of study quality were conducted independently by two researchers using Cochrane Collaboration's tool for assessing risk of bias. PROSPERO registration: CRD42018112732.

Results Twenty-nine studies ( $n=2514$ patients) were included. Nineteen studies compared CT to clinical examination with relative difference in incidence rates ranging from 0.64 to $3.0(n=1369)$. Overall, $79 \%$ of studies found an increase in incidence rate when using CT. Disagreement between CT and clinical examination ranged between 0 and $37.3 \%$ with pooled inter-modality agreement Kappa value of 0.64 (95\% CI 0.52-0.77). Four studies investigated the diagnostic accuracy of ultrasonography $(n=$ 103). Compared with peroperative diagnosis, $\mathrm{CT}$ and ultrasonography both seemed accurate imaging modalities with a sensitivity of $83 \%$.

Conclusion CT is an accurate diagnostic modality for PSH diagnosis and increases PSH detection rates, as compared with clinical examination. Studies that specially focus on the diagnostic accuracy are needed and should aim to take patient-reported outcomes into account. A detailed description of the diagnostic approach, modality, definition, and involved observers is prerequisite for future PSH research.
\end{abstract}

Keywords Parastomal $\cdot$ Hernia $\cdot$ Diagnosis $\cdot$ Modality $\cdot$ Definition $\cdot$ Classification

Electronic supplementary material The online version of this article (https://doi.org/10.1007/s00384-019-03499-5) contains supplementary material, which is available to authorized users.

Gijs H. J. de Smet

g.h.j.desmet@erasmusmc.nl

1 Department of Surgery, Erasmus University Medical Center, 3000 CA Rotterdam, the Netherlands

2 Department of Surgery, IJsselland Ziekenhuis, Capelle aan den IJssel, the Netherlands

3 Department of Surgery, Reinier de Graaf Gasthuis, Delft, the Netherlands

4 Department of Neuroscience, Erasmus University Medical Center, Rotterdam, the Netherlands

\section{Introduction}

Parastomal hernia (PSH) is a common complication following stoma formation and can cause discomfort, pain, strangulation, and incarceration of intestines, as well as difficulties with stoma care [1]. The exact incidence of PSH remains unclear, but most studies report high rates of over $30 \%$, especially in case of colostomy $[1,2]$. Still, reported rates vary widely in the literature, ranging from 0 to $86 \%[1,3,4]$. This variability depends on several factors such as the length of follow-up, patient and surgical characteristics including type of stoma, method of stoma construction, but also on definition of PSH [5-8].

Moreover, several different diagnostic modalities can be used for the diagnosis of PSH, making it a factor affecting the incidence rate. In practice, clinical examination is the first method to assess the presence or absence of a PSH. In case of 
doubt about the diagnosis or to help plan for the surgical approach and management, an imaging modality can be chosen, such as ultrasonography (US), computed tomography (CT) scan, or magnetic resonance imaging (MRI) scan.

In addition, the lack of a clear definition and the use of several different classifications of PSH is a significant problem in PSH research [9]. Some studies use imaging to confirm the diagnosis of PSH, whereas others only use imaging in clinically unconvincing cases $[10,11]$. Due to these differences, protocols often deviate between clinical practice and the research setting, as well as between clinical studies.

In 2014, the European Hernia Society (EHS) proposed a classification depending on the defect size and the presence of a concomitant incisional hernia $[9,12]$. With the ability to correctly compare different studies and thus to provide a uniform research reporting, this classification is recommended by the EHS to use in PSH research [9]. However, these guidelines also emphasize the uncertainties on the accuracy of clinical and imaging diagnoses of PSH.

Therefore, the aim of this systematic review is to evaluate the accuracy of the different modalities used to identify PSH after stoma construction or after PSH repair. The secondary objective is to assess the inter-observer variation, correlations between (a) symptomatic PSH and imaging or surgical findings, and identify different definitions and classifications used for diagnosis of PSH.

\section{Methods}

The study protocol was registered in PROSPERO (CRD42018112732; International Prospective Register of Systematic Reviews). The Preferred Reporting Items for Systematic Reviews and Meta-Analyses of Diagnostic Test Accuracy (PRISMA-DTA) statement was followed [13]. Moreover, the article by Wille-Jørgensen et al. on systematic reviews and meta-analyses in coloproctology was used for methodological guidance [14].

\section{Systematic literature search}

A systematic search was performed by a biomedical information specialist instructed by first author (G.S.). Embase, MEDLINE, Cochrane, Web of Science, and Google Scholar databases were searched on March 5, 2019. Full search strategies and results per database are presented in Appendix 1. There was no limit on date of publication. After duplicate removal, studies were reviewed independently by two researchers (D.L. and G.S.) on title and abstract, followed by full-text review using EndNote X9®. Differences in article selection were discussed, and articles were included or excluded after consensus was reached.

\section{Inclusion and exclusion criteria}

Studies were included if they met the following criteria: (1) inclusion of patients that underwent stoma construction (ileal conduit, ileo- or colostomy) or PSH repair surgery; (2) studies assessing the performance of a diagnostic modality (clinical examination, CT, US, MRI, or diagnosis at surgery) used for the diagnosis of PSH. Only (non) randomized controlled trials, prospective, or retrospective cohort or case-control studies were included. Excluded were as follows: studies reporting on pediatric patients $(<18$ years of age), studies reporting only on gastro-/oesophago- or duodenostomies, studies in which no data on diagnostic modalities were described, and studies with unclear diagnostic work-up, so that diagnostic data could not be extracted. Studies not written in English, case reports, letters, comments, abstracts, or posters were also excluded.

\section{Data extraction}

Data from included studies were extracted by one researcher (G.S.) and were checked independently by another researcher (S.H.) using standard forms covering study characteristics (year, journal, study design, level of evidence, and risk of bias), patient characteristics (number of patients, sex, age, body-mass index, and follow-up), surgical characteristics (indication for surgery, acute or elective, laparoscopic or open abdominal surgery, reoperation, stoma type, use of mesh, location of mesh), and outcome characteristics (definition and classification of PSH, diagnostic modalities and corresponding incidence of PSH and inter-observer variation). Since there is no gold standard modality for diagnosing a PSH, the detection rates of the different diagnostic modalities are compared within each study. The available absolute data and incidence rates of modalities are presented and compared in contingency tables. Intraclass correlation coefficient and Kappa values for interobserver variation were extracted and presented. Intermodality agreements were expressed as Cohen's Kappa values for each study if possible. Statistical level of agreement per Cohen's Kappa value range is presented in Supplemental table 1. The pooled Cohen's Kappa value was calculated in a random effects model using inverse variance method, using meta-package for $\mathrm{R}$ version 3.5.1. (R Foundation for Statistical Computing, Vienna, Austria).

\section{Study quality assessment}

Two researchers (S.H. and G.S.) independently assessed the quality of included studies by assessing the level of evidence 
according to the Oxford Centre for Evidence-based Medicine Levels of Evidence [15] and the possible risk of bias using the Cochrane Collaboration's tool for assessing risk of bias [16] and the QUADAS-2 tool [17] with RevMan 5.3 (Cochrane Centre, Denmark).

\section{Results}

\section{Search and study characteristics}

A PRISMA flow diagram of the complete search results is shown in Fig. 1. After removal of duplicates, 1495 articles were screened on title and abstract of which 192 articles were selected for full-text reading. Finally, 29 articles were judged eligible and were included.

An overview of study characteristics is shown in Table 1. The methodological quality of all included studies per outcome measure is summarized in Fig. 2. Overall, a high risk of bias was present in the included studies (Fig. 3). Applicability concerns were present in 10-20\% of the review sample (Fig. 3). Specific methodological concerns per included study are outlined in Appendix 2 Table 8.

\section{Definition and classification of PSH}

The definition of PSH was reported in eighteen $(62 \%)$ of the included studies $[2,11,19-22,25,27,28,31$, 33, 34, 37, 39, 41-43]. Some studies used two different definitions for clinical and radiologic examination [20, 21, 27, 31]. Therefore, a total of nineteen different definitions were used (Appendix 3 Table 9). For the definitions used in clinical examination, most studies included a combination of the terms "bulge" or "protrusion" and "around" or "in the vicinity of" the stoma. Also, some studies added the position of the patient's body (supine or/and erect) during examination and the use of the Valsalva maneuver. For the definitions used in radiological examination, the terms "defect," "fascia," and "hernia sac" were often incorporated in the definition. Five studies did not describe the definition of PSH or diagnostic approach [18, 23, 24, 29, 35].
Fig. 1 Preferred items for reporting of systematic reviews and meta-analyses (PRRISMA) flow diagram

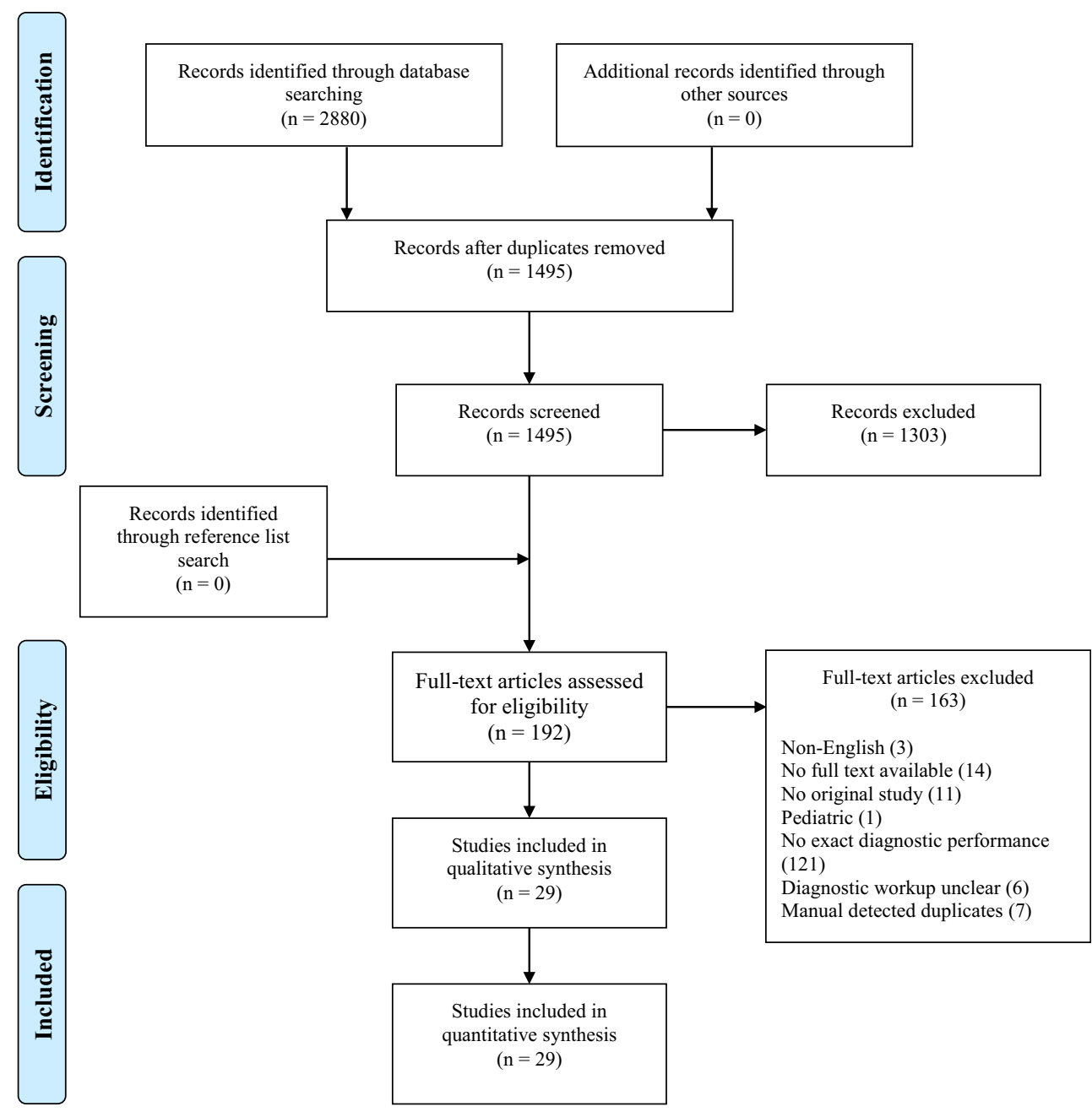







Fig. 2 Risk of bias and applicability concerns summary

\begin{tabular}{|c|c|c|c|c|c|c|c|c|}
\hline & & & isk c & f Bia & & Appli & cabili & ty $\mathrm{Co}$ \\
\hline & & 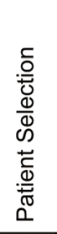 & 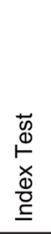 & 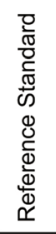 & 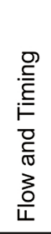 &  & 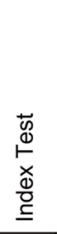 & 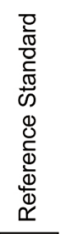 \\
\hline Inter-observer variation & Gurmu 2011 & + & + & & + & + & + & \\
\hline & Jänes 2011 & + & + & & + & + & + & \\
\hline & Strigard 2013 & - & $?$ & & + & + & + & \\
\hline CT-scan vs clinical & Aslam 2019 & + & $\odot$ & $?$ & + & + & + & $?$ \\
\hline & Brandsma 2017 & 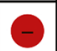 & + & + & $\theta$ & $\theta$ & + & + \\
\hline & Canda 2018 & 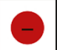 & 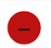 & $?$ & + & + & + & + \\
\hline & Cingi 2006 & + & $?$ & $?$ & $\Theta$ & + & + & + \\
\hline & Conde-Muino 2017 & + & + & + & + & + & + & + \\
\hline & Etherington 1990 & + & $\theta$ & + & + & + & + & + \\
\hline & Fleshman 2014 & - & $\odot$ & $?$ & 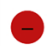 & $\odot$ & + & + \\
\hline & Hauters 2016 & + & $?$ & $?$ & + & $\odot$ & + & + \\
\hline & Hino 2017 & + & $?$ & + & + & + & + & + \\
\hline & Hong 2013 & + & $\odot$ & $?$ & + & $\odot$ & $?$ & + \\
\hline & Ihnát 2018 & $?$ & + & + & + & + & + & + \\
\hline & Köhler 2016 & - & $?$ & $?$ & $\Theta$ & $?$ & + & + \\
\hline & Lambrecht 2015 & + & + & + & + & + & + & + \\
\hline & Moreno-Matias 2009 & + & + & + & + & + & + & + \\
\hline & Näsvall 2017 & - & $?$ & $?$ & $\odot$ & + & - & + \\
\hline & Nikberg 2015 & - & $?$ & $?$ & 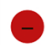 & + & ? & + \\
\hline & Odensten 2017 & - & + & + & . & + & + & + \\
\hline & Seo 2011 & + & + & $?$ & $?$ & + & + & $?$ \\
\hline & Serra-Aracil 2009 & + & + & $?$ & + & + & + & + \\
\hline & Timmermans 2014 & $O$ & $?$ & $?$ & ) & $\theta$ & + & + \\
\hline & Veirimaa 2015 & + & $?$ & + & + & + & + & + \\
\hline $\begin{array}{l}\text { Ultrasonography vs } \\
\text { clinical examination }\end{array}$ & Sjödahl 2011 & + & + & + & + & + & + & + \\
\hline $\begin{array}{l}\text { CT-scan vs } \\
\text { ultrasonography }\end{array}$ & Näsvall 2014 & + & $?$ & $?$ & + & + & + & + \\
\hline Imaging vs clinical & Donahue 2014 & - & + & $O$ & $?$ & + & + & + \\
\hline & Hansson 2013 & - & $?$ & + & 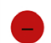 & - & + & + \\
\hline & Hotouras 2013 & 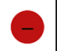 & $?$ & $?$ & ) & - & $?$ & $?$ \\
\hline Peroperative diagnosis & Näsvall 2014 & + & + & + & + & + & + & + \\
\hline & Fleshman 2014 & - & - & - & & - & $?$ & $?$ \\
\hline gh & ? Unclear & & & & & & & \\
\hline
\end{tabular}




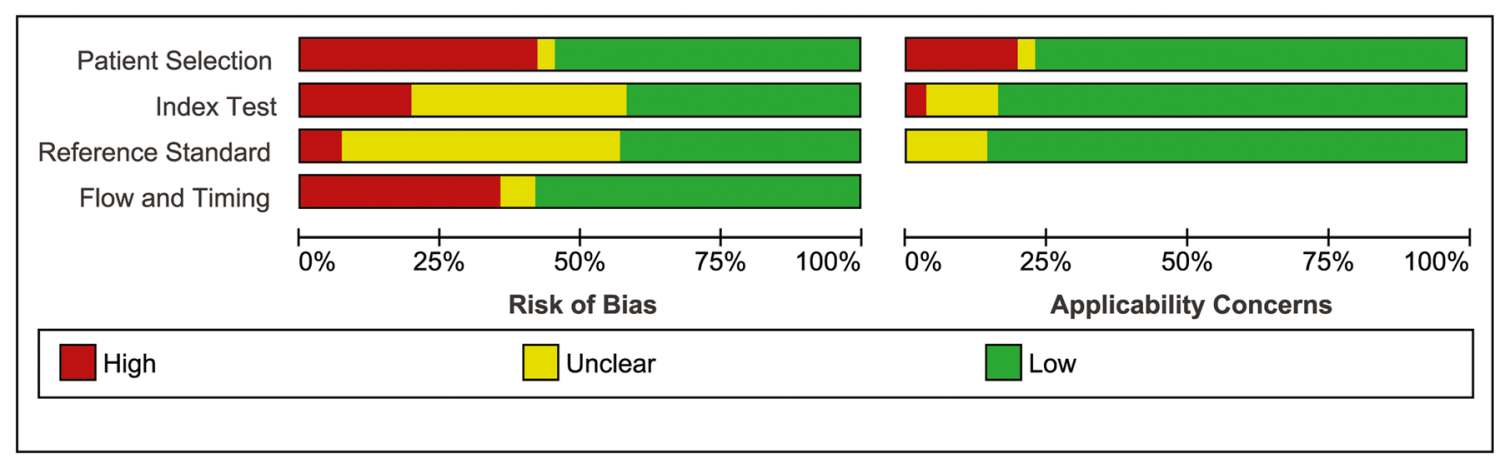

Fig. 3 Overall risk of bias and applicability concerns

The classification of PSH was reported in thirteen $(45 \%)$ of the included studies $[2,10,22,26,30$, 32-34, 36, 38-40, 43]. Two classifications were used. One developed and introduced by the European Hernia Society [12] and one by Moreno-Matias [34] (Appendix 4 Table 10).

\section{Inter-observer variation}

Three of the included studies reported on inter-observer variation $[25,31,42]$. Each study investigated different modalities examined by different observers. An overview of the methods and results of these studies is summarized in Table 2. Gurmu et al. reported a low inter-observer reliability when diagnosing PSH by clinical examination with disagreement rates of 35 and $54 \%$ between three surgeons and $18 \%$ between two surgeons [25]. Jänes et al. reported a strong agreement between three surgeons after diagnosing PSH by clinical examination with a Kappa value of 0.85 [31]. Also, the inter-observer reliability was higher among radiologists when patients underwent a CT in prone position as compared with patients in supine position with Kappa values of 0.85 and 0.82 , respectively [31]. Strigård et al. investigated inter-observer reliability and learning curve of three-dimensional ultrasonography (3D US) in 40 patients. They found an overall interobserver agreement of $72 \%$ with a Kappa value of 0.59 , which is classified as "weak." The learning curve reached its top at around 30 patients with an inter-observer agreement of $80 \%$ for the last ten examined patients [42].

\section{CT versus clinical examination}

The incidence rates of PSH after CT and clinical examination were reported in nineteen studies including a total of 1369 patients $[2,18-21,23,26-28,30,32$, 33, 36-39, 43]. PSH incidence rates, disagreement percentages, and Kappa values are presented in Table 3. Study quality and clinico-radiological concordance are presented in Supplemental table 2. Fifteen studies (79\%) reported a higher incidence rate and two studies (11\%) reported lower incidence rate when diagnosing PSH using CT as compared with clinical diagnoses. When comparing CT to clinical examination, the relative difference in incidence rates ranged from 0.64 to

Table 2 Inter-observer variation

\begin{tabular}{|c|c|c|c|c|c|}
\hline \multirow[t]{4}{*}{ Gurmu [25] } & Risk of bias & +++ & & Disagreement $(\%)$ & Kappa \\
\hline & Level of evidence & $2 b$ & Hospital I, 3 surgeons, $n=17$ & 35 & $0.35-0.64$ \\
\hline & \multirow{2}{*}{\multicolumn{2}{|c|}{ Panel of 5 surgeons, clinical examination ${ }^{\mathrm{a}}$}} & Hospital II, 3 surgeons, $n=13$ & 54 & $0.29-0.43$ \\
\hline & & & Hospital III, 2 surgeons, $n=11$ & 18 & 0.73 \\
\hline \multirow[t]{6}{*}{ Jänes [31] } & Risk of bias & +++ & $N=27$ & & Kappa \\
\hline & Level of evidence & $2 b$ & 3 Surgeons, clinical examination & & 0.85 \\
\hline & \multirow{4}{*}{\multicolumn{2}{|c|}{6 Observers: 3 surgeons and 3 radiologists }} & 3 Radiologists, $\mathrm{CT}$ in prone pos & & 0.85 \\
\hline & & & 3 Radiologists, $\mathrm{CT}$ in supine po & & 0.82 \\
\hline & & & Surgeons and radiologists collec & $\mathrm{T}$ in prone position & 0.80 \\
\hline & & & Surgeons and radiologists collec & $\mathrm{T}$ in supine position & 0.64 \\
\hline \multirow[t]{4}{*}{ Strigård [42] } & Risk of bias & + & & Disagreement $(\%)$ & Kappa \\
\hline & Level of evidence & $2 b$ & 3 Investigators, $n=17$ & 41 & $\sim$ \\
\hline & \multirow{2}{*}{\multicolumn{2}{|c|}{ Panel of 3 physicians, 3D ultrasonography }} & 2 Investigators, $n=40$ & 20 & $\sim$ \\
\hline & & & Combined & 28 & 0.59 \\
\hline
\end{tabular}

${ }^{\mathrm{a}}$ Choosing between: hernia, bulge or no hernia 
Table 3 CT versus clinical examination

\begin{tabular}{|c|c|c|c|c|c|c|c|}
\hline Study & $N$ & $\begin{array}{l}\text { Incidence CT } \\
\text { vs CE (\%) }\end{array}$ & $\begin{array}{l}\text { Relative increase } \\
\text { with CT }\end{array}$ & $\begin{array}{l}\text { Disagreement CE } \\
\text { vs CT }(\%)\end{array}$ & Kappa value & Standard Error & $95 \% \mathrm{CI}$ \\
\hline Aslam [18] & 17 & CT $18 \%$, CE $18 \%$ & 1 & 0 & 1 & 0.00 & $1.00-1.00$ \\
\hline Canda [19] & 67 & CT $28 \%$, CE $22 \%$ & 1.27 & 6 & 0.84 & 0.08 & $0.69-0.99$ \\
\hline Cingi [20] & 21 & CT $86 \%$, CE $52 \%$ & 1.5 & 28.6 & 0.36 & 0.22 & $0.00-0.79$ \\
\hline Conde-Muino [21] & 31 & CT $7 \%$, CE $3 \%$ & 2 & 3.2 & 0.65 & 0.34 & $0.00-1.00$ \\
\hline Etherington [23] & 28 & CT $36 \%$, CE $29 \%$ & 1.25 & 11.1 & 0.84 & 0.11 & $0.62-1.00$ \\
\hline Hauters [26] & 29 & CT $7 \%$, CE $3 \%$ & 2 & 3.5 & 0.65 & 0.34 & $0.00-1.00$ \\
\hline Hino [27] & 59 & CT $17 \%$, CE $20 \%$ & 0.93 & 37.3 & 0.25 & 0.13 & $0.00-0.50$ \\
\hline Hong [28] & 108 & CT $27 \%$, CE $33 \%$ & 1.24 & 6.5 & 0.85 & 0.06 & $0.74-0.96$ \\
\hline Köhler [32] & 51 & CT $4 \%$, CE $1 \%$ & 3 & 3.9 & 0.49 & 0.36 & $0.00-1.00$ \\
\hline Lambrecht [33] & 58 & CT $33 \%$, CE $24 \%$ & 1.36 & 22.4 & 0.45 & 0.13 & $0.19-0.72$ \\
\hline Moreno-Matias [34] & 75 & CT $47 \%$, CE $44 \%$ & 1.06 & 29.3 & 0.41 & 0.11 & $0.20-0.62$ \\
\hline Näsvall [36] & 47 & CT $15 \%$, CE $22 \%$ & 0.64 & 17 & 0.46 & 0.17 & $0.11-0.80$ \\
\hline Seo [39] & 83 & CT $29 \%$, CE $24 \%$ & 1.2 & 4.8 & 0.88 & 0.06 & $0.76-0.99$ \\
\hline Serre-Aracil [40] & 54 & CT $33 \%$, CE $28 \%$ & 1.2 & 5.56 & 0.87 & 0.07 & $0.73-1.00$ \\
\hline Veirimaa [2] & 67 & CT $49 \%$, CE $25 \%$ & 1.94 & 23.9 & 0.52 & 0.10 & $0.31-0.72$ \\
\hline Fleshman [24] & $\sim$ & CT $11 \%$, CE $13 \%$ & $\sim$ & $\sim$ & & & \\
\hline Ihnát [30] & 148 & CT $53 \%$, CE $48 \%$ & $\sim$ & $\sim$ & & & \\
\hline Nikberg [37] & $187(141 \mathrm{CT})$ & CT $53 \%$, CE $25 \%$ & $\sim$ & $\sim$ & & & \\
\hline Odensten [38] & $211(198 \mathrm{CT})$ & CT $35 \%$, CE $29 \%$ & $\sim$ & $\sim$ & & & \\
\hline Timmermans [43] & $150(87 \mathrm{CT})$ & CT $53 \%$, CE $53 \%$ & $\sim$ & $\sim$ & & & \\
\hline \multicolumn{5}{|c|}{ Pooled Kappa value, random effects model } & 0.64 & & $0.52-0.77$ \\
\hline
\end{tabular}

$C E$, clinical examination; $C T$, computed tomography scan

3.0. Disagreement between diagnoses by using CT versus clinical examination could be obtained in fifteen studies and ranged from 0 to $37.3 \%$. The pooled intermodality agreement Kappa value for all fourteen studies with contingency tables was 0.64 (95\% CI $0.52-0.77)$ which is classified as "substantial agreement."

\section{Ultrasonography versus clinical examination}

The incidence rates of PSH after US and clinical examination were reported in one study, which included 43 patients with peristomal bulging (Table 4, Supplemental table 3) [41]. Sjödahl et al. reported a lower incidence rate by US for diagnosing PSH with relative difference of 0.58 when compared with clinical examination. The disagreement between these modalities was $53.5 \%$.

\section{CT versus ultrasonography}

Studies comparing PSH incidence of CT to regular US were not identified. One study by Näsvall et al. [35] investigated intrastomal 3D US as an alternative to CT and included twenty patients that were indicated for surgical revision due to stoma-related symptoms. The PSH incidence was higher when using CT (80\%) as compared with 3D US (75\%) (Table 5, Supplemental table 4).

\section{Peroperative diagnosis}

Näsvall et al. compared 3D US and CT to findings at surgery in twenty patients [35]. For both imaging modalities a high sensitivity of $83 \%$ was found. A positive predictive value (PPV) of $94 \%$ and a negative predicted value (NPV) of $75 \%$ were reported for diagnosis with CT. For diagnosis with 3D US, a PPV of $100 \%$ and a

Table 4 Ultrasonography versus clinical examination

\begin{tabular}{lllllll}
\hline Study & $N$ & Incidence US vs CE (\%) & Relative increase with US & Disagreement CE vs US (\%) & Kappa value & Standard error \\
\hline Sjödahl [41] & 43 & US 35\%, CE 61\% & 0.58 & $53.5 \%$ & -0.01 & 0.94 \\
\hline
\end{tabular}

CE, clinical examination; US, ultrasonography 
Table 5 CT versus ultrasonography

\begin{tabular}{lllll}
\hline Study & $N$ & Incidence CT vs 3D US (\%) & $\begin{array}{l}\text { Relative increase } \\
\text { with US }\end{array}$ & $\begin{array}{l}\text { Disagreement } \\
\text { CT vs US (\%) }\end{array}$ \\
\hline Nasväll [35] & 20 & CT 80\%, US 75\% & $\sim$ & $\sim$ \\
\hline
\end{tabular}

$C T$, computed tomography scan; $U S$, ultrasonography
NPV of $60 \%$ were reported. Also, Fleshman et al. reported peroperative findings in thirteen patients who were diagnosed with PSH at clinical examination of which eleven were confirmed by CT and two were confirmed operatively [24]. Study quality, PSH incidence rates, and surgico-radiological concordance of the two studies are presented in Table 6 and Supplemental table 5.

\section{Imaging versus clinical examination}

Two studies reported on clinical examination, CT and MRI for the diagnosis of PSH. These studies did not subdivide the incidence rate per type of imaging modality [11,22]. Study quality, PSH incidence rates, and clinico-radiological concordance of the studies are presented in Table 7 and Supplemental table 6. Donahue et al. reported a higher incidence rate when using imaging with a relative increase of 1.47 and found no patients with clinical detected but radiological occult PSH [22]. Hansson et al. found three symptomatic PSHs in 60 patients that were clinically examined. A CT or MRI was performed in 27 of the 60 patients of whom nineteen patients had a asymptomatic hernia. Hotouras et al. reported 25 (58\%) PSHs diagnosed with CT. Eleven (44\%) of these 25 patients with radiological confirmed PSH were symptomatic as reported by the patients.

\section{Imaging after clinical suspicion of parastomal hernia}

Brandsma et al. and Fleshman et al. used only a CT when there was clinical suspicion of PSH. In the study of Brandsma et al. [10], sixteen out of nineteen clinical PSHs (14.3\%) were confirmed by CT, two by MRI, and one by US. Fleshman et al. found thirteen $(13 \%)$ clinical PSHs of which eleven (11\%) were confirmed by $\mathrm{CT}$ and two peroperatively. Hansson et al. performed a CT or MRI when there were doubts about the diagnosis of PSH during clinical examination [11]. One participating center performed imaging routinely (Table 7). The incidence after clinical examination was 5\% (3/60) and after imaging $7 \%(4 / 61)$.

\section{Discussion}

Today, in both clinical practice and research there is no gold standard modality to examine patients for the presence of PSH. The literature on this subject is diverse and inconclusive. Facilitating comparison between studies on PSH remains challenging, due to, among others, the number of existing definitions, imaging modalities, and classifications. Indeed, this systematic review shows a great variance in detection rates of PSH between different diagnostic modalities.

Most included studies compared CT with clinical examination. The majority of these studies found higher incidence rates by using CT $[2,19-21,23,26,28,30,32-34,37-40]$. However, some studies showed contradictory results in favor of clinical examination $[24,27,36]$. This discrepancy between studies could be explained by the technical differences in examination of the patients' abdominal wall, bearing in mind that a patients' body position and the use of Valsalva maneuver during examination might affect detection rates [31]. It is possible to use Valsalva maneuver in case of patients undergoing CT imaging. However, this is rarely reported in studies.

Gurmu et al. found a low inter-observer reliability when patients were clinically examined by surgeons, indicating that PSH is difficult to diagnose by clinical examination [25]. This was also stated by Sjödahl et al. who found poor correlation between US and findings at clinical examination [41]. If these examinations are performed correctly, the use of dynamic modalities such as US and clinical examination may have some advantages compared with the more static and expensive CT or MRI. However, the inter-observer variation and diagnostic accuracy of US have not been investigated thoroughly. In contrast, more evidence is available on the diagnostic performance of clinical examination and CT. For research purposes, the combined use of these two modalities might be recommended since multiple studies found significant disagreements in detection rates between both modalities [27, 33, 34].

Table 6 Peroperative diagnosis

\begin{tabular}{lllll}
\hline Study & $N$ & Incidence CT vs surgery (\%) & Incidence US vs surgery (\%) & $\begin{array}{c}\text { Disagreement imaging } \\
\text { vs surgery (\%) }\end{array}$ \\
\hline Nasväll [35] & 20 & CT 80\%, surgery 90\% & $\sim$ & $20 \%$ \\
Fleshman [24] & $\sim$ & CT 11\%, surgery 13\% & $\sim$ & $\sim$ \\
Nasväll [35] & 20 & $\sim$ & US 75\%, surgery 90\% & $15 \%$ \\
\hline
\end{tabular}

$C T$, computed tomography scan; $U S$, ultrasonography 
Table 7 Imaging versus clinical examination

\begin{tabular}{llllll}
\hline Study & $N$ & Incidence imaging vs CE (\%) & $\begin{array}{l}\text { Relative increase } \\
\text { with imaging }\end{array}$ & $\begin{array}{l}\text { Disagreement } \\
\text { imaging vs CE (\%) }\end{array}$ & Kappa value \\
\hline Donahue [22] & 386 & Imaging 36\%, CE 24\% & 1.47 & 15 & 0.73 \\
\hline
\end{tabular}

$C E$, clinical examination

This is the first review to date that provides a complete overview of the research of the available literature on different diagnostic modalities for PSH diagnosis. Nevertheless, it is important to note that this systematic review covers studies that investigate the PSH incidence rates in the setting of a research protocol that might not always fully reflect standard clinical practice. Also, the minority of included studies has the accuracy of the used diagnostic modality as primary outcome [20, 23, 25, 31, 34, 35, 41, 42]. In clinical practice the main goal is to identify symptomatic PSHs that might require treatment and for asymptomatic patients it seems unnecessary to follow a full diagnostic workup. Therefore, the clinical approach might differ from that in a research setting. In general, patients with stoma problems such as pain, appliance leakage, bowel obstruction, or symptoms of incarceration first undergo clinical examination by a stoma nurse and/or clinician. When PSH is identified clinically or the diagnosis is inconclusive the clinician can consider an imaging modality to confirm the diagnosis, taking into account patient safety, patient comfort, availability, and costs, whereas for research purposes, factors as costs and availability might play a less important role in the decision on imaging modality.

Intrastomal 3D US is a relatively new imaging modality for diagnosing PSH or other stoma-related pathology [44]. 3D US seems to be an accurate imaging modality with a sensitivity of $83 \%$ when compared with peroperative diagnosis [35]. With this imaging modality it is possible to examine patients in erect position and without the use of radiation, providing potential advantages over CT. There is, however, too little available evidence for this technique to consider this as standard imaging modality for the diagnosing of PSH.

In contrast to diagnosing incisional hernia, traditional twodimensional ultrasonography (2D US) is not often used for diagnosing PSH in both research and in clinical setting. However, 2D US is the most patient-friendly, inexpensive, and practical modality of all imaging modalities. This systematic review included only one study comparing 2D US to clinical examination for diagnosing PSH. However, to make any recommendations on 2D US, it would be interesting to compare ultrasonography with other imaging modalities in the future.

Another important aspect of clinical practice with regard to the use of diagnostic modalities is that many stoma patients have a stoma created after oncological resection, and for these patients a CT is routinely made during follow-up to detect potential cancer recurrence. Although some PSHs occur many years after stoma construction, most PSHs develop within the first years after stoma construction and are thus likely to be identified with follow-up CT [5]. This is one of the main reasons why most included studies used $\mathrm{CT}$ instead of MRI or US. However, with the patient in supine position a CT is not a reliable tool for diagnosing $\mathrm{PSH}$ and a CT with the patient in prone position is associated with higher inter-observer agreement and an increase in sensitivity [31]. By using CT routinely for cancer follow-up, asymptomatic PSHs will appear more frequently. Although not entirely insignificant, studies do not often distinguish between symptomatic and asymptomatic when reporting PSH incidence rates.

Evidently, patient-reported outcomes are of paramount importance in the context of stoma-related complications. Patients know their own bodies in a way no physician possibly can, and have to take care of the stoma several times a day, whereas the physician examines the patients' stoma once or maybe twice. Any physical differences of the stoma will be noticed by the patient, which probably makes it more reliable than the studied modalities on the existence of bulging at some time point during follow-up. Currently, prospective cohort studies, such as the PROPHER and CIPHER studies (ISRCTN17573805; ISRCTN registry), are assessing the value of subjective and objective outcomes after stoma construction or for parastomal hernia treatment, respectively.

Despite the increased interest in PSH care and research in recent decades, there is still no consensus regarding the definition of PSH or a gold standard for diagnosis [9]. Although many definitions consisted of similar terms and contexts, some definitions differ considerably which can lead to discrepancies in detection rates. Moreover, the fact that five included studies have not even described the definition of PSH, emphasizes the need for uniform reporting in studies regarding PSH [18, 23, 24, 29, 35]. This heterogeneity in diagnostic procedures makes it difficult to compare studies and to determine an accurate incidence of PSH. Therefore, a clear and standard definition and diagnosis of PSH is of paramount importance. The European Hernia Society (EHS) acknowledged this problem and proposed to use the definition of PSH introduced by Muysoms et al. [45]: "An incisional hernia through the abdominal wall defect created during placement of a colostomy, ileostomy or ileal conduit stoma". Furthermore, the EHS proposed a new classification for PSH, which might help to facilitate more uniform reporting of outcomes in PSH research (Appendix 4 Table 10) [12]. 


\section{Limitations}

This systematic review has some limitations. Firstly, the low level of evidence of included studies is an important limitation. Eleven studies have a retrospective study design, which is prone for selection and information bias. Also, most studies presented small study populations. Nevertheless, to give a complete overview of diagnostic accuracy and variation of the different modalities, studies of low quality or studies with small samples were not excluded and a comprehensive overview of study characteristics and study quality assessment was provided.

Secondly, significant heterogeneity between studies was demonstrated, as operation and stoma types, use of mesh reinforcement, patient characteristics (e.g., age and BMI), and follow-up duration differed between included studies. Besides the choice of diagnostic modality, all these factors also influence the PSH incidence rates. Although it was not possible to account for this, these factors would be of less importance for the within-study diagnostic performance, since diagnostic modalities were only compared within each study. However, some studies did not investigate the PSH incidence rate or the accuracy of the diagnostic modalities as primary objective. As a result, the incidence could easily be underestimated. Accordingly, the results of diagnostic performance may also be affected.

\section{Conclusion}

In conclusion, this review shows great variance in accuracy of different modalities for the detection of PSH. The use of CT increases the PSH detection rate, indicating that this is a more accurate modality compared with clinical examination. However, the evidence on the accuracy of the other imaging modalities, also within patient-reported outcome measures, is scarce and warrants further investigation. There are significant differences in diagnostic methods between clinical practice and in the setting of research protocols, as well as between clinical studies. In order to compare studies correctly and increase transparency among studies, a more detailed report of the diagnostic method and a detailed and preferably uniform definition are required in future research. It might be of added value to develop a standard and validated protocol in which self-report, clinical examination, and imaging are combined.

Acknowledgments The authors thank Wichor Bramer, biomedical information specialist at the Erasmus Medical Center, for his assistance with the search strategy and syntax.

\section{Compliance with ethical standards}

Conflict of interest The authors declare that they have no conflicts of interest.

\section{Appendix 1. Literature search syntax}

\section{Embase.com}

('parastomal hernia'/exp. OR ((stoma/exp. OR 'enterostomy'/ exp) AND ('abdominal wall hernia'/de OR hernia/de OR hernioplasty/de OR 'herniorrhaphy'/de)) OR (((parastoma* OR stoma OR stomal OR colostom* OR ileostom* OR jejunostom* OR parajejunostom* OR cecostom* OR paracecostom* OR duodenostom* OR paraduodenostom* OR urostom* OR paracolostom* OR paraileostom* OR paraurostom*) NEAR/6 (hernia* OR hernioplast* OR herniorrha*))):ab,ti) NOT ([Conference Abstract]/lim OR [Letter]/lim OR [Note]/lim OR [Editorial]/lim) AND [english]/lim.

\section{Medline Ovid}

(((exp Surgical Stomas/ OR exp. Enterostomy/) AND (Hernia, Ventral/ OR Hernia, Abdominal/ OR Hernia/ OR Herniorrhaphy/)) OR (((parastoma* OR stoma OR stomal OR colostom* OR ileostom* OR jejunostom* OR parajejunostom* OR cecostom* OR paracecostom* OR duodenostom* OR paraduodenostom* OR urostom* OR paracolostom* OR paraileostom* OR paraurostom*) ADJ6 (hernia* OR hernioplast* OR herniorrha*))).ab,ti.) NOT (letter OR news OR comment OR editorial OR congresses OR abstracts).pt. AND english.la.

\section{Cochrane CENTRAL}

((((parastoma* OR stoma OR stomal OR colostom* OR ileostom* OR jejunostom* OR parajejunostom* OR cecostom* OR paracecostom* OR duodenostom* OR paraduodenostom* OR urostom* OR paracolostom* OR paraileostom* OR paraurostom*) NEAR/6 (hernia* OR hernioplast* OR herniorrha*))):ab,ti)

\section{Web of Science}

$\mathrm{TS}=((((($ parastoma* OR stoma OR stomal OR colostom* OR ileostom* OR jejunostom* OR parajejunostom* OR cecostom* OR paracecostom* OR duodenostom* OR paraduodenostom* OR urostom* OR paracolostom* OR paraileostom* OR paraurostom*) NEAR/5 (hernia* OR hernioplast* OR herniorrha*))))) AND LA = (english) AND $\mathrm{DT}=($ article $)$ 


\section{Google scholar}

"parastomal|stomal|stoma|colostomal|ileostomal|jejunostoma1|parajejunostomal|cecostomal|paracecostomal|duodenostom- al|paraduodenostomal|urostomal|paracolostomal|paraileostomal|paraurostomal hernia"

\section{Appendix 2}

Table 8 Methodological concerns

Inter-observer variation

Gurmu

Jänes

Strigård

CT-scan vs clinical examination

Brandsma

Canda

Cingi

Conde-Muino

Etherington

Fleshman

Hauters

Hino

Hong

Köhler

Lambrecht

Moreno-Matias

Näsvall 2017

Nikberg

Odensten

Seo

Serra-Aracil

Timmermans

Veirimaa
Selection of patients depending on different hospitals (selection bias); no definition used for clinical examination (reporting bias)

No major methodological concerns

One experienced physicians and two with short training

Only a CT-scan was performed when there was a clinical suspicion of a PSH (selection bias)

Patients with no postoperative available CT-scan were excluded (selection bias); no definition used for clinical examination (reporting bias)

Unclear whether comparison was blinded (reporting bias)

No major methodological concerns

No definition used for imaging (reporting bias)

Only a CT-scan was performed when there was a clinical suspicion of a PSH (selection bias); no definition used (reporting bias)

No definition used for clinical examination (reporting bias)

Unclear whether comparison was blinded (reporting bias)

Unclear whether comparison was blinded (reporting bias); no definition used for clinical examination (reporting bias)

Not all included patients underwent a CT-scan (selection bias); no definition used for clinical examination (reporting bias)

No major methodological concerns

No major methodological concerns

Part of included patient did not underwent imaging (selection bias); Unclear whether comparison was blinded (reporting bias)

Part of included patient did not underwent imaging (selection bias); Unclear whether comparison was blinded (reporting bias)

Part of included patient did not underwent imaging (selection bias)

Interval between CT-scan and clinical examination unclear

No definition used for clinical examination (reporting bias)

Part of included patient did not underwent imaging (selection bias); Interval between CT-scan and clinical examination unclear

Unclear whether comparison was blinded (reporting bias)

Ultrasonography vs clinical examination

Sjödahl

No major methodological concerns

CT-scan vs ultrasonography

Näsvall $2014 \quad$ No definition used (reporting bias)

Imaging vs clinical examination

Donahue

No definition used for clinical examination (reporting bias); unclear whether all patients underwent clinical examination (reporting bias)

Hansson

Only in clinical unconvincing cases a CT-scan or a MRI was performed (selection bias); in one of the participating center a CT-scan or MRI was performed routinely (selection bias)

Hotouras

Patients with no postoperative available CT-scan were excluded (selection bias); only symptomatic or asymptomatic PSHs were reported. No clinical examination was reported (reporting bias)

Peroperative diagnosis

Fleshman No definition used (reporting bias)

Näsvall $2014 \quad$ No major methodological concerns 


\section{Appendix 3}

Table 9 Definition of parastomal hernia

\begin{tabular}{|c|c|}
\hline Study & Definition of parastomal hernia \\
\hline Strigård & 'Defect of the fascia with a protruding hernia sac at the passage of the stoma intestine through the abdominal wall' \\
\hline Veirimaa & $\begin{array}{l}\text { 'Clinically significant parastomal hernia was defined here as parastomal hernia associated with stoma appliance dysfunction } \\
\text { and leakage not responsive to conservative measures, peristomal skin breakdown related to sheer injury or ischemia from } \\
\text { pressure on the thinned peristomal skin, and recurrent partial bowel obstruction' }\end{array}$ \\
\hline \multicolumn{2}{|c|}{ Definition clinical examination } \\
\hline Cingi & 'Bulging during the Valsalva maneuver and palpation of the fascial defect' \\
\hline Conde-Muino & 'Any noticeable bulge, in the vicinity of the ostomy with the patient erect, supine, and performing the Valsalva maneuver' \\
\hline Hansson & $\begin{array}{l}\text { 'Recurrent or persistent bulge when the patient is standing during a Valsalva maneuver, or palpation of the fascial defect with } \\
\text { the patient in the supine position' }\end{array}$ \\
\hline Hino & 'Any protrusion around the stoma observed during physical examination' \\
\hline Jänes & 'Any protrusion in the vicinity of the stoma with the patient straining in a supine and an erect position' \\
\hline Lambrecht & 'Bulge associated with the stoma' \\
\hline Seo & 'Any protrusion in the vicinity of the stoma' \\
\hline Sjödahl & $\begin{array}{l}\text { 'A wide opening (more than two fingers) presenting as a manifest parastomal hernia with a palpable bowel segment or } \\
\text { omentum passing through the abdominal opening together with the stoma bowel' }\end{array}$ \\
\hline Timmermans & $\begin{array}{l}\text { 'Any palpable defect or bulge adjacent to the stoma when the patient was supine with their elevated legs or erect and coughing } \\
\text { or straining' }\end{array}$ \\
\hline \multicolumn{2}{|l|}{ Definition imaging } \\
\hline $\begin{array}{l}\text { Canda, Conde-Muino, } \\
\text { Jänes }\end{array}$ & 'Any intraabdominal content protruding beyond the peritoneum or the presence of a hernia sac' \\
\hline $\begin{array}{l}\text { Donahue, } \\
\text { Moreno-Matias }\end{array}$ & 'The protrusion of abdominal contents through the abdominal wall defect created by forming the stoma' \\
\hline Cingi & $\begin{array}{l}\text { 'A loop of intestine or any abdominal organ, as well as preperitoneal fat, protruding through the defect alongside the ostomy } \\
\text { was considered as parastomal hernia' }\end{array}$ \\
\hline Gurmu & 'A defect in the fascia through which intraabdominal contents such as omentum or bowel could be extruded out' \\
\hline Hino & $\begin{array}{l}\text { '(1) Herniation of a loop of intestine other than the distal colon, (2) sliding and winding of the distal colon, or (3) herniation of } \\
\text { any structures such as the omentum and a winder defect of the parastomal abdominall wall fascia' }\end{array}$ \\
\hline Hong & 'Any intraabdominal content protruding beyond the fascia or the presence of a hernia sac' \\
\hline Näsvall 2017 & 'A peritoneal sac protruding through the fascia beside the stoma bowel' \\
\hline Nikberg & 'Any intra-abdominal content protruding beyond the peritoneum or the presence of a hernia sac at least 1 year after operation' \\
\hline
\end{tabular}

\section{Appendix 4}

Table 10 Classification of parastomal hernia

\begin{tabular}{|c|c|c|c|}
\hline Study & Developed by & \multicolumn{2}{|c|}{ Description classification } \\
\hline \multirow[t]{6}{*}{$\begin{array}{l}\text { Brandsma, Köhler, Lambrecht, } \\
\text { Näsvall 2017, Timmermans, Vierimaa }\end{array}$} & \multirow[t]{6}{*}{$\begin{array}{l}\text { European Hernia } \\
\text { Society }\end{array}$} & & Primary \\
\hline & & & Recurrence \\
\hline & & Type 1 & $<5 \mathrm{~cm}$ \\
\hline & & Type 2 & $<5 \mathrm{~cm}$, concomitant incisional hernia \\
\hline & & Type 3 & $>5 \mathrm{~cm}$ \\
\hline & & Type 4 & $>5 \mathrm{~cm}$, concomitant incisional hernia \\
\hline \multirow{5}{*}{$\begin{array}{l}\text { Brandsma, Donahue, Hauters, Köhler, } \\
\text { Lambrecht, Moreno-Matias, Odensten, } \\
\text { Seo, Serra-Aracil }\end{array}$} & \multirow[t]{5}{*}{ Moreno-Matias } & Type 0 & $\begin{array}{l}\text { Peritoneum follows the wall of the bowel forming the stoma, with } \\
\text { no formation of a sac }\end{array}$ \\
\hline & & Type 1a & Bowel forming the colostomy with a sac $<5 \mathrm{~cm}$ \\
\hline & & Type $1 \mathrm{~b}$ & Bowel forming the colostomy with a sac $>5 \mathrm{~cm}$ \\
\hline & & Type 2 & Sac containing omentum \\
\hline & & Type 3 & Intestinal loop other than the bowel forming the stoma \\
\hline
\end{tabular}


Open Access This article is licensed under a Creative Commons Attribution 4.0 International License, which permits use, sharing, adaptation, distribution and reproduction in any medium or format, as long as you give appropriate credit to the original author(s) and the source, provide a link to the Creative Commons licence, and indicate if changes were made. The images or other third party material in this article are included in the article's Creative Commons licence, unless indicated otherwise in a credit line to the material. If material is not included in the article's Creative Commons licence and your intended use is not permitted by statutory regulation or exceeds the permitted use, you will need to obtain permission directly from the copyright holder. To view a copy of this licence, visit http://creativecommons.org/licenses/by/4.0/.

\section{References}

1. Carne PW, Robertson GM, Frizelle FA (2003) Parastomal hernia. Br J Surg 90(7):784-793

2. Vierimaa M, Klintrup K, Biancari F, Victorzon M, CarpelanHolmstrom M, Kossi J et al (2015) Prospective, randomized study on the use of a prosthetic mesh for prevention of parastomal hernia of permanent colostomy. Dis Colon Rectum 58(10):943-949

3. Shabbir J, Chaudhary BN, Dawson R (2012) A systematic review on the use of prophylactic mesh during primary stoma formation to prevent parastomal hernia formation. Color Dis 14(8):931-936

4. Ho KK, Economou T, Smart NJ, Daniels IR (2019) Radiological progression of end colostomy trephine diameter and area. BJS Open 3(1):112-118

5. Mylonakis E, Scarpa M, Barollo M, Yarnoz C, Keighley MR (2001) Life table analysis of hernia following end colostomy construction. Color Dis 3(5):334-337

6. Sohn YJ, Moon SM, Shin US, Jee SH (2012) Incidence and risk factors of parastomal hernia. J Korean Soc Coloproctol 28(5):241246

7. Sjodahl R, Anderberg B, Bolin T (1988) Parastomal hernia in relation to site of the abdominal stoma. Br J Surg 75(4):339-341

8. Kroese LF, de Smet GHJ, Jeekel J, Kleinrensink GJ, Lange JF (2016) Systematic review and meta-analysis of extraperitoneal versus transperitoneal colostomy for preventing parastomal hernia. Dis Colon Rectum 59(7):688-695

9. Antoniou SA, Agresta F, Garcia Alamino JM, Berger D, Berrevoet F, Brandsma HT, Bury K, Conze J, Cuccurullo D, Dietz UA, Fortelny RH, Frei-Lanter C, Hansson B, Helgstrand F, Hotouras A, Jänes A, Kroese LF, Lambrecht JR, Kyle-Leinhase I, LópezCano M, Maggiori L, Mandalà V, Miserez M, Montgomery A, Morales-Conde S, Prudhomme M, Rautio T, Smart N, Śmietański M, Szczepkowski M, Stabilini C, Muysoms FE (2018) European Hernia Society guidelines on prevention and treatment of parastomal hernias. Hernia. 22(1):183-198

10. Brandsma HT, Hansson BM, Aufenacker TJ, van Geldere D, Lammeren FM, Mahabier C, Makai P, Steenvoorde P, de Vries Reilingh TS, Wiezer MJ, de Wilt JH, Bleichrodt RP, Rosman C, Dutch Prevent Study group (2017) Prophylactic mesh placement during formation of an end-colostomy reduces the rate of parastomal hernia: short-term results of the Dutch PREVENT-trial. Ann Surg 265(4):663-669

11. Hansson BM, Morales-Conde S, Mussack T, Valdes J, Muysoms FE, Bleichrodt RP (2013) The laparoscopic modified Sugarbaker technique is safe and has a low recurrence rate: a multicenter cohort study. Surg Endosc 27(2):494-500

12. Smietanski M, Szczepkowski M, Alexandre JA, Berger D, Bury K, Conze J et al (2014) European Hernia Society classification of parastomal hernias. Hernia. 18(1):1-6
13. McInnes MDF, Moher D, Thombs BD, McGrath TA, Bossuyt PM, and the P-DTAG et al (2018) Preferred reporting items for a systematic review and meta-analysis of diagnostic test accuracy studies: the PRISMA-DTA statement. JAMA. 319(4):388-396

14. Wille-Jorgensen P, Renehan AG (2008) Systematic reviews and meta-analyses in coloproctology: interpretation and potential pitfalls. Color Dis 10(1):21-32

15. Howick J, Chalmers I., Glasziou, P., Greenhalgh, T., Heneghan C., Liberati, A., Moschetti, I., Phillips, B., Thornton, H., Goddard, O., Hodgkinson, M. Oxford Centre for Evidence-Based Medicine. The Oxford 2011 Levels of Evidence. [Available from: http://www. cebm.net/index.aspx?o=5653. Accessed 22 Nov 2018

16. Higgins JP, Altman DG, Gøtzsche PC, Jüni P, Moher D, Oxman $\mathrm{AD}$ et al (2011) The Cochrane Collaboration's tool for assessing risk of bias in randomised trials. BMJ. 343:d5928

17. Whiting PF, Rutjes AW, Westwood ME, Mallett S, Deeks JJ, Reitsma JB, Leeflang MM, Sterne JA, Bossuyt PM, QUADAS-2 Group (2011) QUADAS-2: a revised tool for the quality assessment of diagnostic accuracy studies. Ann Intern Med 155(8):529-536

18. Aslam MI, Baloch N, Mann C, Nilsson PJ, Maina P, Chaudhri S et al (2019) Simultaneous stoma reinforcement and perineal reconstruction with biological mesh - a multicentre prospective observational study. Ann Med Surg (Lond) 38:28-33

19. Canda AE, Terzi C, Agalar C, Egeli T, Arslan C, Altay C, Obuz F (2018) Preventing parastomal hernia with modified stapled mesh stoma reinforcement technique (SMART) in patients who underwent surgery for rectal cancer: a case-control study. Hernia. 22(2):379-384

20. Cingi A, Cakir T, Sever A, Aktan AO (2006) Enterostomy site hernias: a clinical and computerized tomographic evaluation. Dis Colon Rectum 49(10):1559-1563

21. Conde-Muino R, Diez JL, Martinez A, Huertas F, Segura I, Palma P (2017) Preventing parastomal hernias with systematic intraperitoneal specifically designed mesh. BMC Surg 17(1):41

22. Donahue TF, Bochner BH, Sfakianos JP, Kent M, Bernstein M, Hilton WM, Cha EK, Yee AM, Dalbagni G, Vargas HA (2014) Risk factors for the development of parastomal hernia after radical cystectomy. J Urol 191(6):1708-1713

23. Etherington RJ, Williams JG, Hayward MW, Hughes LE (1990) Demonstration of para-ileostomy herniation using computed tomography. Clin Radiol 41(5):333-336

24. Fleshman JW, Beck DE, Hyman N, Wexner SD, Bauer J, George V et al (2014) A prospective, multicenter, randomized, controlled study of non-cross-linked porcine acellular dermal matrix fascial sublay for parastomal reinforcement in patients undergoing surgery for permanent abdominal wall ostomies. Dis Colon Rectum 57(5): 623-631

25. Gurmu A, Matthiessen P, Nilsson S, Pahlman L, Rutegard J, Gunnarsson U (2011) The inter-observer reliability is very low at clinical examination of parastomal hernia. Int J Color Dis 26(1):89 95

26. Hauters P, Cardin JL, Lepere M, Valverde A, Cossa JP, Auvray S, Framery D, Zaranis C (2016) Long-term assessment of parastomal hernia prevention by intra-peritoneal mesh reinforcement according to the modified Sugarbaker technique. Surg Endosc 30(12):53725379

27. Hino H, Yamaguchi T, Kinugasa Y, Shiomi A, Kagawa H, Yamakawa Y, Numata M, Furutani A, Suzuki T, Torii K (2017) Relationship between stoma creation route for end colostomy and parastomal hernia development after laparoscopic surgery. Surg Endosc 31(4):1966-1973

28. Hong SY, Oh SY, Lee JH, Kim DY, Suh KW (2013) Risk factors for parastomal hernia: based on radiological definition. J Korean Surg Soc 84(1):43-47

29. Hotouras A, Murphy J, Power N, Williams NS, Chan CL (2013) Radiological incidence of parastomal herniation in cancer patients 
with permanent colostomy: what is the ideal size of the surgical aperture? Int J Surg 11(5):425-427

30. Ihnat P, Tulinsky L, Jonszta T, Koscielnik P, Ihnat Rudinska L, Penka I 2018 Parastomal and incisional hernia following laparoscopic/open abdominoperineal resection: is there a real difference? Surg Endosc.

31. Janes A, Weisby L, Israelsson LA (2011) Parastomal hernia: clinical and radiological definitions. Hernia. 15(2):189-192

32. Kohler G, Hofmann A, Lechner M, Mayer F, Wundsam H, Emmanuel K et al (2016) Prevention of parastomal hernias with $3 \mathrm{D}$ funnel meshes in intraperitoneal onlay position by placement during initial stoma formation. Hernia. 20(1):151-159

33. Lambrecht JR, Larsen SG, Reiertsen O, Vaktskjold A, Julsrud L, Flatmark K (2015) Prophylactic mesh at end-colostomy construction reduces parastomal hernia rate: a randomized trial. Color Dis 17(10):O191-O197

34. Moreno-Matias J, Serra-Aracil X, Darnell-Martin A, BombardoJunca J, Mora-Lopez L, Alcantara-Moral M, Rebasa P, Ayguavives-Garnica I, Navarro-Soto S (2009) The prevalence of parastomal hernia after formation of an end colostomy. A new clinico-radiological classification. Color Dis 11(2):173-177

35. Nasvall P, Wikner F, Gunnarsson U, Rutegard J, Strigard K (2014) A comparison between intrastomal 3D ultrasonography, CT scanning and findings at surgery in patients with stomal complaints. Int $\mathbf{J}$ Color Dis 29(10):1263-1266

36. Nasvall P, Rutegard J, Dahlberg M, Gunnarsson U, Strigard K (2017) Parastomal hernia repair with intraperitoneal mesh. Surg Res Pract 2017:8597463

37. Nikberg M, Sverrisson I, Tsimogiannis K, Chabok A, Smedh K (2015) Prophylactic stoma mesh did not prevent parastomal hernias. Int J Color Dis 30(9):1217-1222

38. Odensten C, Strigard K, Rutegard J, Dahlberg M, Stahle U, Gunnarsson U et al (2017) Use of prophylactic mesh when creating a colostomy does not prevent parastomal hernia: a randomized controlled trial-STOMAMESH. Ann Surg

39. Seo SH, Kim HJ, Oh SY, Lee JH, Suh KW (2011) Computed tomography classification for parastomal hernia. J Korean Surg Soc 81(2):111-114

40. Serra-Aracil X, Bombardo-Junca J, Moreno-Matias J, Darnell A, Mora-Lopez L, Alcantara-Moral M, Ayguavives-Garnica I, Navarro-Soto S (2009) Randomized, controlled, prospective trial of the use of a mesh to prevent parastomal hernia. Ann Surg 249(4):583-587

41. Sjodahl RI, Thorelius L, Hallbook OJ (2011) Ultrasonographic findings in patients with peristomal bulging. Scand J Gastroenterol 46(6):745-749

42. Strigard K, Gurmu A, Nasvall P, Pahlman P, Gunnarsson U (2013) Intrastomal 3D ultrasound; an inter- and intra-observer evaluation. Int J Color Dis 28(1):43-47

43. Timmermans L, Deerenberg EB, Lamme B, Jeekel J, Lange JF (2014) Parastomal hernia is an independent risk factor for incisional hernia in patients with end colostomy. Surgery. 155(1):178-183

44. Gunnarsson U, Strigard K (2012) 3D intrastomal ultrasonography, an instrument for detecting stoma-related fistula. Tech Coloproctol 16(3):233-236

45. Muysoms F, Campanelli G, Champault GG, DeBeaux AC, Dietz UA, Jeekel J, Klinge U, Köckerling F, Mandala V, Montgomery A, Morales Conde S, Puppe F, Simmermacher RK, Śmietański M, Miserez M (2012) EuraHS: the development of an international online platform for registration and outcome measurement of ventral abdominal wall hernia repair. Hernia. 16(3):239-250

Publisher's note Springer Nature remains neutral with regard to jurisdictional claims in published maps and institutional affiliations. 